MARIJA PERIĆ

Sveučilište u Zadru

nmiletic2@unizd.hr

mperic5@unizd.hr

\title{
HEILIGENNAMEN IN TOURISTISCHEN BROSCHÜREN UND REISEFÜHRERN VON ZADAR
}

S chlüs s e lw ört e r: Heiligennamen, touristische Reiseführer und Broschüren, Endonyme, Exonyme

\section{EINFÜHRUNG}

Onomastik oder Namenkunde ist die Kunst des Namengebens, d.h. die Wissenschaft über Namen als sprachliche, außersprachliche und vor allem kulturelle Denkmäler. Namen werden in unterschiedlichen Wissenschaften erforscht, weil sie mit ihrem Wortinhalt und ihrer Wortgestalt sprachliche und außersprachliche Informationen über Raum und Zeit vermitteln. Deswegen ist der Name für Wissenschaftler (z.B. Geschichte, Geographie, Archäologie, Ethnologie u.a.) ein peripherer Gegenstand, aber kein Kern der Forschung. Da der Name vor allem ein sprachliches Zeichen ist, gehört Onomastik zur Sprachwissenschaft, obwohl nicht alles, was Onomastik erforscht, Forschungsgebiet der Sprachwissenschaft ist (vgl. Duden 2003: 1164; Frančić 2015: 75; Šimunović 2009: 15).

R. Matasović (1997: 91) schlägt strukturelle und etymologische methodologische Kriterien für die Namenforschung vor. Das strukturelle Kriterium ist: der Name $N$ gehört zur Sprache $L$, wenn die Regeln $P_{1} \ldots P_{\mathrm{n}}$ auf N wie auch auf andere Elemente der Sprache $L$ angewendet werden. Das etymologische Kriterium ist: der Name $N$ gehört zur Sprache $L$, wenn es innerhalb der Sprache $L$ eine etymologische Verbindung mit den Elementen, die in $L$ eine Bedeutung haben, gibt. Diese beiden Kriterien beziehen sich gleichermaßen auf Antroponyme und Toponyme, mit der Anmerkung, dass auch die strengsten methodologischen Kriterien eine fehlerhafte etymologische Beurteilung nicht verhindern können, wenn sprachhistorische Fakten nicht in Betracht gezogen werden.

Namen haben verschiedene Funktionen. Sie können zur Orientierung in einer Umgebung dienen (Toponyme), zur Kommunikation mit anderen Menschen (Antroponyme) und zur Bezeichnung der materiellen und nicht-materiellen Produkte (Krematonyme) (vgl. Frančić 2015: 75). Neben diesen Termini bezeichnet P. Šimunović (2009: 15) Toponyme als Geonyme und Antroponyme als 
Bionyme, während er auch hervorhebt, dass immer neue Termini in diesem Bereich entstehen. Demzufolge führt er Nesonyme als Namen der Inseln, Potamonyme als Namen der Flüsse, Hagionyme als Heiligennamen und Eklezionyme als Namen von Patronen der Kirchen an.

In diesem Beitrag werden Hagionyme und Eklezionyme im Zusammenhang mit Kirchennamen aus Zadar erforscht. Deswegen ist es notwendig, die Termini Endonym und Exonym zu erklären.

Exonyme sind Ortsnamenformen, die in anderen Ländern gebraucht werden und sich von amtlichen Namen, die als Endonyme bezeichnet werden, unterscheiden, z.B. Gdańsk ist der Name einer Stadt in Polen, wobei sie außerhalb Polens als Danzig bekannt ist, weil Geographen meinen, dass Exonym weltweit bekannter als das Endonym ist. Der Begriff Exonym ist noch nicht präzise definiert und wird nicht als ein Terminus systematisch verwendet. Es hat eine mehrdeutige Konnotation und kann mehrere Namenarten bezeichnen (vgl. Duden 2003: 504; Harder 1995: 1012). Demzufolge werden in diesem Beitrag die Heiligennamen bzw. auch die Namen der Kirchen mit den Termini Exonym und Endonym bezeichnet.

\section{METHODOLOGIE, ZIEL UND FORSCHUNGSFRAGEN}

Für diesen Beitrag wurden die Reiseführer und Broschüren von Zadar analysiert. Die Reiseführer, die analysiert wurden, sind kroatische, englische und deutsche Reiseführer von Travirka und Petricioli. In diesen Reiseführern und Broschüren wurden alle vorhandenen Namen der Heiligen, die in Zadar geehrt werden, extrahiert und interlingual verglichen, da Unregelmäßigkeiten in der Verwendung der Namen von Heiligen in den erwähnten Reiseführern und Broschüren bemerkt wurden.

Die Fragen, die beantwortet werden, sind folgende: Wird ein Name systematisch verwendet oder kommt in mehreren Varianten vor? Werden in den englischsprachigen und deutschsprachigen Reiseführern und Broschüren endonymische oder exonymische Varianten der Namen verwendet?

Für die Analyse wurden englischsprachige und deutschsprachige Reiseführer von A.Travirka aus den Jahren 1998 und 2010 und von I. Petricioli aus dem Jahr 1997 recherchiert, während für die Ausgangssprache nur ein kroatischsprachiger Reiseführer von A. Travirka (2003) und ein von I. Petricioli (2001) analysiert wurde. Die Broschüren, die analysiert wurden, sind: otkrij... Zadar, discover ... Zadar und entdecke... Zadar (2016). Um einen Vorschlag des Gebrauchs der Namen der Heiligen auszuarbeiten, wurden die Heiligennamen in Printwörterbüchern und auf Internetseiten überprüft. Die Printwörterbücher, die benutzt wurden, sind MikićGojmerac (2011): Religionswörterbuch Kroatisch-Deutsch (2005), A Dictionary of Jewish Christian Relations und Hitchcock (1869): Hitchcock's Bible Names 
Dictionary. Die Internetseiten, auf denen die Namen aufgesucht wurden, sind www. catholic.org und www.americancatholic.org ${ }^{1}$.

\section{ANALYSE DER HEILIGENNAMEN IN TOURISTISCHEN REISEFÜHRERN UND BROSCHÜREN}

In Zadar werden vier Schutzpatronen geehrt: Sv. Stošija, Sv. Šime, Sv. Krševan und Sv. Zoilo. Neben diesen vier Namen werden zusätzlich 21 Heiligennamen erforscht, bei denen einige Unregelmäßigkeiten bemerkt wurden.

\subsection{Krševan}

In beiden kroatischen Reiseführern wird der Name Krševan verwendet. In englischen Reiseführern von Travirka werden Krševan, Grisogonos (Krševan), Krševan (Grisogonus), Grisogonus und Chrysogonus angeführt. Den Unterschied zwischen den Versionen von 1998 und 2010 bildet der Name Chrysogonus, der nur in der Version aus dem Jahre 2010 vorkommt. In Petriciolis Reiseführer wird das Endonym Krševan verwendet, während nur einmal das Exonym Chrysogone in Klammern erscheint.

In deutschen Reiseführern von Travirka werden Grisogonus, Chrysogonos, Chrysogonos (Krševan) und Grisogonus (Kř̌evan) verwendet. In der neueren Ausgabe aus dem Jahr 2010 wird Chrysogonos angeführt, wobei er in der älteren Ausgabe fehlt. In Petriciolis Reiseführer wurde nur das Exonym Grisogonus systematisch verwendet.

Tabelle 1. Travirka - Krševan

\begin{tabular}{|c|c|c|c|c|c|c|c|c|c|}
\hline \multirow{2}{*}{\multicolumn{2}{|c|}{$\frac{\text { KROATISCH }}{2003}$}} & \multicolumn{4}{|c|}{ ENGLISCH } & \multicolumn{4}{|c|}{ DEUTSCH } \\
\hline & & \multicolumn{2}{|l|}{1998} & \multicolumn{2}{|l|}{2010} & \multicolumn{2}{|l|}{1998} & \multicolumn{2}{|l|}{2010} \\
\hline Krševan & 12 & Krševan & 8 & Krševan & 4 & & & & \\
\hline & & $\begin{array}{c}\text { Grisogonos } \\
\text { (Krševan) }\end{array}$ & 1 & $\begin{array}{c}\text { Grisogonos } \\
\text { (Krševan) }\end{array}$ & 1 & & & $\begin{array}{c}\text { Grisogonus } \\
\text { (Krsevan) }\end{array}$ & 1 \\
\hline & & $\begin{array}{c}\text { Krševan } \\
\text { (Grisogonus) }\end{array}$ & 1 & $\begin{array}{c}\text { Krševan } \\
\text { (Grisogonus) }\end{array}$ & 1 & & & $\begin{array}{c}\text { Chrysogonos } \\
\text { (Krševan) }\end{array}$ & 1 \\
\hline & & Grisogonus & 3 & Grisogonus & 3 & Grisogonus & 9 & Grisogonus & 5 \\
\hline & & & & Chrysogonus & 8 & & & Chrysogonos & 7 \\
\hline
\end{tabular}

1 Im weiteren Text werden folgende Abkürzungen genutzt: Mikić-Gojmerac 2011 — MG; KesslerWenborn 2005 — KW; Hitchcock 1869 - H; www.catholic.org — URL1 und www.americancatholic. org - URL2. 
Tabelle 2. Petricioli - Krševan

\begin{tabular}{|c|c|c|r|c|c|}
\hline \multicolumn{2}{|c|}{ KROATISCH -2001 } & \multicolumn{2}{c|}{ ENGLISCH - 1997 } & \multicolumn{2}{c|}{ DEUTSCH-1997 } \\
\hline Krševan & 18 & Krševan & 17 & Grisogonus & 17 \\
\hline & & Krševan (Chrysogone) & 1 & & \\
\hline
\end{tabular}

In Broschüren erscheinen folgende Namen: KRO Krševan - ENG Grisogone - DE Grisogonus.

In den Wörterbüchern MG, KW, H und auf URL2 wurden keine Varianten des Namens gefunden, während URL1 nur das Exonym Chrysogonus anführt.

\subsection{Stošija (Anastazija)}

Tabelle 3. Travirka - Stošija (Anastazija)

\begin{tabular}{|c|c|c|c|c|c|c|c|c|c|}
\hline \multirow{2}{*}{\multicolumn{2}{|c|}{$\begin{array}{c}\text { KROATISCH } \\
2003\end{array}$}} & \multicolumn{4}{|c|}{ ENGLISCH } & \multicolumn{4}{|c|}{ DEUTSCH } \\
\hline & & \multicolumn{2}{|c|}{1998} & \multicolumn{2}{|l|}{2010} & \multicolumn{2}{|l|}{1998} & \multicolumn{2}{|l|}{2010} \\
\hline & & $\begin{array}{c}\text { Anastasia } \\
\text { (Stošija) }\end{array}$ & 1 & $\begin{array}{c}\text { Anastasia } \\
(\text { Stošija })\end{array}$ & 1 & $\begin{array}{c}\text { Anastasia } \\
(\text { Stošija })\end{array}$ & 1 & $\begin{array}{c}\text { Anastasia } \\
(\text { Stosija })\end{array}$ & 1 \\
\hline $\begin{array}{c}\text { Stošija } \\
\text { (Anastazija) }\end{array}$ & 2 & & & $\begin{array}{c}\text { Stošija } \\
\text { (Anastasia) }\end{array}$ & 2 & & & & \\
\hline Stošija & 9 & Stošija & 3 & Stošija & 3 & & & Anastasia & 9 \\
\hline Anastazija & 1 & Anastasia & 2 & Anastasia & 5 & Anastasia & 8 & Anasthasia & 2 \\
\hline
\end{tabular}

Tabelle 4. Petricioli — Stošija (Anastazija)

\begin{tabular}{|c|c|c|c|c|c|}
\hline \multicolumn{2}{|c|}{ KROATISCH -2001 } & \multicolumn{2}{c|}{ ENGLISCH - 1997 } & \multicolumn{2}{c|}{ DEUTSCH - 1997 } \\
\hline Stošija & 6 & & & Anastasia & 12 \\
\hline Anastazija & 1 & Stošija (Anastasia) & 4 & Anastasia (Stosija) & 1 \\
\hline Stošija (Anastazija) & 4 & Stošija & 6 & & \\
\hline
\end{tabular}

In beiden kroatischen Reiseführern kommen die Varianten Stošija und Anastazija vor. In allen englischen Reiseführern werden die Varianten Anastasia und Stošija abwechselnd verwendet.

In Travirkas und Petriciolis deutschen Reiseführern wird der Name Anastasia präferiert, während das Endonym Stošija nur einmal erscheint. Der Name Anastasia wird aber in Travirkas Reiseführer aus dem Jahr 2010 mit zwei lexikographischen Varianten Anastasia und Anasthasia geschrieben.

In Broschüren erscheinen folgende Namen: KRO Stošija - ENG Anastasia DE Anastasija.

In den Wörterbüchern MG, KW und $\mathrm{H}$ wurden keine Varianten des Namens gefunden, während URL1 nur das Exonym Anastasia anführt. 


\section{3. Šime}

Tabelle 5. Travirka — Šime

\begin{tabular}{|c|c|c|c|c|c|c|c|c|c|}
\hline \multicolumn{2}{|c|}{ KROATISCH } & \multicolumn{3}{|c|}{ ENGLISCH } & \multicolumn{3}{c|}{ DEUTSCH } \\
\hline \multicolumn{2}{|c|}{2003} & \multicolumn{2}{|c|}{1998} & \multicolumn{2}{c|}{2010} & \multicolumn{2}{c|}{1998} & \multicolumn{2}{c|}{2010} \\
\hline Šimun & 18 & Simeon & 17 & Simeon & 24 & Simeon & 19 & Simeon & 23 \\
\hline Šime & 9 & & & & & & & & \\
\hline
\end{tabular}

Tabelle 6. Petricioli — Šme

\begin{tabular}{|c|c|c|c|c|r|}
\hline \multicolumn{2}{|c|}{ KROATISCH - 2001 } & \multicolumn{2}{|c|}{ ENGLISCH - 1997 } & \multicolumn{2}{c|}{ DEUTSCH - 1997 } \\
\hline Šimun & 14 & Šimun (Simeon) & 1 & Simeon & 20 \\
\hline & & Šimun & 18 & Simeon (Simun) & 1 \\
\hline
\end{tabular}

Im kroatischen Reiseführer von Travirka werden abwechselnd die Varianten Šimun und Šime verwendet, während in dem Reiseführer von Petricioli nur der Name Šimun erscheint.

In Travirkas englischen und deutschen Reiseführern wird nur der Name Simeon systematisch verwendet, während sich Petricioli für die englische Variante mit dem Endonym Šimun und für die deutsche Version mit dem Exonym Simeon entscheidet. In Petriciolis englischsprachigem Reiseführer wird die exonymische Variante Simeon nur einmal erwähnt, und im deutschsprachigen Reiseführer wird die endonymische Variante Šimun nur einmal angeführt.

In Broschüren erscheinen folgende Namen: KRO Šime - ENG Simon - DE Simeon.

Im Wörterbuch MG wird der Name Šimun ins Deutsche als Simeon übersetzt, während die Variante Šime nicht erwähnt wird. Im Wörterbuch H und auf URL1 wird der Name Simeon erwähnt, während im Wörterbuch KW keine Variante des Namens gefunden wurde.

\subsection{Zoilo}

Tabelle 7. Travirka — Zoilo

\begin{tabular}{|c|c|c|c|c|c|c|c|c|}
\hline \multicolumn{2}{|c|}{ KROATISCH } & \multicolumn{3}{|c|}{ ENGLISCH } & \multicolumn{3}{c|}{ DEUTSCH } \\
\hline \multicolumn{2}{|c|}{2003} & \multicolumn{2}{|c|}{1998} & \multicolumn{2}{c|}{2010} & \multicolumn{2}{c|}{2098} & \multicolumn{2}{c|}{ Zoilo } & 2 \\
\hline
\end{tabular}

Tabelle 8. Petricioli — Zoilo

\begin{tabular}{|c|c|c|c|c|c|}
\hline \multicolumn{2}{|c|}{ KROATISCH -2001 } & \multicolumn{2}{|c|}{ ENGLISCH - 1997 } & \multicolumn{2}{|c|}{ DEUTSCH - 1997 } \\
\hline Zoilo & 1 & Zoilo & 3 & Zoilo & 3 \\
\hline
\end{tabular}

Im kroatischsprachigen Reiseführer von Travirka wird Zoilo nicht erwähnt, während er in Petricioli erscheint. 
In englischsprachigen Reiseführern von Travirka wird der Name Zoilus nur in der neueren Ausgabe angeführt, während Petricioli die endonymische Variante Zoilo erwähnt.

In deutschsprachigen Reiseführern bedienen sich Travirka und Petricioli des Endonyms Zoilo.

In den Broschüren wird der Heiligenname Zoilo nicht angeführt.

In den Wörterbüchern MG, KW und H wurden keine Varianten des Namens gefunden, während URL1 das Exonym Zoilus anführt.

\subsection{Donat}

Tabelle 9. Travirka — Donat

\begin{tabular}{|c|c|c|c|c|c|c|c|c|c|}
\hline \multirow{2}{*}{\multicolumn{2}{|c|}{$\begin{array}{c}\text { KROATISCH } \\
2003\end{array}$}} & \multicolumn{4}{|c|}{ ENGLISCH } & \multicolumn{4}{|c|}{ DEUTSCH } \\
\hline & & \multicolumn{2}{|c|}{1998} & \multicolumn{2}{|c|}{2010} & \multicolumn{2}{|c|}{1998} & \multicolumn{2}{|c|}{2010} \\
\hline Donat & 11 & Donat & 9 & Donat & 7 & & & & \\
\hline & & Donatus & 2 & Donatus & 3 & Donatus & 9 & Donatus & 8 \\
\hline
\end{tabular}

Tabelle 10. Petricioli - Donat

\begin{tabular}{|c|c|c|c|c|c|}
\hline \multicolumn{2}{|c|}{ KROATISCH - 2001 } & \multicolumn{2}{|c|}{ ENGLISCH - 1997 } & \multicolumn{2}{c|}{ DEUTSCH - 1997 } \\
\hline Donat & 9 & Donat & 9 & Donat & 7 \\
\hline & & & & Donatus & 2 \\
\hline
\end{tabular}

In kroatischsprachigen Reiseführern wird nur das Endonym Donat verwendet. In beiden englischsprachigen Reiseführern werden die Varianten Donat und Donatus abwechselnd benutzt.

In Travirkas deutschsprachigen Reiseführern wird das Exonym Donatus systematisch verwendet, während sich Petricioli abwechselnd der Varianten Donatus und Donat bedient.

In Broschüren erscheinen folgende Namen: KRO Donat - ENG Donat - DE Donat/Donatus.

In den Wörterbüchern MG und KW wurde der Name Donatus gefunden, während auf URL1 beide Varianten des Namens vorkommen. Im Wörterbuch H wurden keine Einträge gefunden.

\subsection{Frane}

Tabelle 11. Travirka - Frane

\begin{tabular}{|c|c|c|c|c|c|c|c|c|c|}
\hline KROATISCH & \multicolumn{5}{|c|}{ ENGLISCH } & \multicolumn{4}{c|}{ DEUTSCH } \\
\hline \multicolumn{2}{|c|}{2003} & \multicolumn{2}{|c|}{1998} & \multicolumn{2}{c|}{2010} & \multicolumn{2}{c|}{1998} & \multicolumn{2}{c|}{2010} \\
\hline Frane & 11 & Francis & 2 & Francis & 10 & Franziskus & 7 & Franciscus & 6 \\
\hline Franjo & 2 & & & & & Franciscus & 4 & Franciscus & 4 \\
\hline & & & & Francis de Sales & 1 & & & & \\
\hline
\end{tabular}


Tabelle 12. Petricioli - Frane

\begin{tabular}{|c|c|c|c|c|c|}
\hline \multicolumn{2}{|c|}{ KROATISCH - 2001 } & \multicolumn{2}{|c|}{ ENGLISCH - 1997 } & \multicolumn{2}{c|}{ DEUTSCH - 1997 } \\
\hline Frane & 2 & Francis & 6 & Franziskus & 6 \\
\hline Franjo & 5 & & & Franziskus (Sveti Frane) & 1 \\
\hline
\end{tabular}

In kroatischsprachigen Reiseführern werden die endonymischen Varianten Frane und Franjo verwendet.

In englischsprachigen Reiseführern wird das Exonym Francis systematisch gebraucht.

In deutschsprachigen Reiseführern von Travirka werden die exonymischen lexikographischen Varianten Franziskus und Franciskus abwechselnd verwendet, während sich Petricioli der exonymischen Variante Franziskus bedient und nur einmal die endonymische Variante Frane benutzt.

In Broschüren erscheinen folgende Namen: KRO Frane - ENG Francis DE Franziskus.

Im Wörterbuch MG wird der Name Franjo als Franz ins Deutsche übersetzt, während das Wörterbuch KW und die Internetseite URL1 die englische Variante Francis nennen. Im Wörterbuch H wurde keine Variante des Namens gefunden.

\subsection{Marija}

Tabelle 13. Travirka - Marija

\begin{tabular}{|c|c|c|c|c|c|c|c|c|c|}
\hline \multirow{2}{*}{\multicolumn{2}{|c|}{$\begin{array}{c}\text { KROATISCH } \\
2003 \\
\end{array}$}} & \multicolumn{4}{|c|}{ ENGLISCH } & \multicolumn{4}{|c|}{ DEUTSCH } \\
\hline & & \multicolumn{2}{|l|}{1998} & \multicolumn{2}{|l|}{2010} & \multicolumn{2}{|l|}{1998} & \multicolumn{2}{|l|}{2010} \\
\hline Marija & 11 & Mary & 12 & Mary & 15 & Maria & 1 & Maria & 5 \\
\hline $\begin{array}{c}\text { Marija } \\
\text { Magdalena }\end{array}$ & 1 & $\begin{array}{c}\text { Mary } \\
\text { Magdalene }\end{array}$ & 1 & $\begin{array}{c}\text { Mary } \\
\text { Magdalene }\end{array}$ & 1 & $\begin{array}{c}\text { Maria } \\
\text { Magdalena }\end{array}$ & 1 & $\begin{array}{c}\text { Maria } \\
\text { Magdalena }\end{array}$ & 1 \\
\hline $\begin{array}{c}\text { Marija } \\
\text { Velika }\end{array}$ & 2 & $\begin{array}{c}\text { Mary } \\
\text { the Great }\end{array}$ & 1 & & & $\begin{array}{c}\text { Maria die } \\
\text { Große } \\
\text { (Mariä } \\
\text { Himmelfahrt) }\end{array}$ & 2 & $\begin{array}{c}\text { Maria } \\
\text { die Große } \\
(\text { Maria } \\
\text { Himmelfahrt })\end{array}$ & 2 \\
\hline \multirow[t]{2}{*}{$\begin{array}{c}\text { Marija } \\
\text { „de } \\
\text { Pusterla“, } \\
\text { Stomorica }\end{array}$} & 1 & $\begin{array}{c}\text { Maria } \\
\text { „,de } \\
\text { Pusterla“, } \\
\text { Stomorica }\end{array}$ & 1 & $\begin{array}{c}\text { Maria } \\
\text {,de Pusterla“, } \\
\text { Stomorica }\end{array}$ & 1 & $\begin{array}{l}\text { Maria die } \\
\text { Pusterla, } \\
\text { genannt } \\
\text { Stomorica }\end{array}$ & 1 & $\begin{array}{c}\text { Maria } \\
\text { „,de Pusterla“, } \\
\text { genannt } \\
\text { Stomorica }\end{array}$ & 1 \\
\hline & & & & $\begin{array}{c}\text { Mary } \\
\text {,de } \\
\text { Pusterla“, } \\
\text { Stomorica }\end{array}$ & 1 & & & & \\
\hline
\end{tabular}


Tabelle 14. Petricioli — Marija

\begin{tabular}{|c|c|c|c|c|c|}
\hline \multicolumn{2}{|c|}{ KROATISCH - 2001 } & \multicolumn{2}{c|}{ ENGLISCH - 1997 } & \multicolumn{2}{c|}{ DEUTSCH - 1997 } \\
\hline Marija & 10 & Mary & 10 & Maria & 6 \\
\hline Marija Velika & 3 & Mary the Great & 1 & $\begin{array}{c}\text { Mariä Himmelfahrt } \\
\text { (Marija Velika) }\end{array}$ & 2 \\
\hline Maria de Pusterla & 2 & $\begin{array}{c}\text { Mary Major } \\
\text { (and the Old) }\end{array}$ & 2 & & \\
\hline
\end{tabular}

In kroatischsprachigen Reiseführern wird das Endonym Marija angeführt, was in den englischsprachigen Reiseführern als Mary und in deutschsprachigen als Maria übersetzt wird.

In Broschüren erscheinen folgende Namen: KRO Marija — ENG Mary — DE Marija/Maria.

Im MG wird Marija als Maria übersetzt. In den Wörterbüchern KW und H wird das Exonym Mary angeführt, während auf URL1 Maria Magdalen und URL2 Virgin Mary und Mary Magdalene verwendet werden.

\subsection{Dominik}

Tabelle 15. Travirka - Dominik

\begin{tabular}{|c|c|c|c|c|c|c|c|c|c|}
\hline \multicolumn{2}{|c|}{ KROATISCH } & \multicolumn{3}{c|}{ ENGLISCH } & \multicolumn{3}{c|}{ DEUTSCH } \\
\hline \multicolumn{2}{|c|}{2003} & \multicolumn{2}{|c|}{1998} & \multicolumn{2}{c|}{2010} & \multicolumn{2}{c|}{1998} & \multicolumn{2}{c|}{2010} \\
\hline Dominik & 7 & Dominic & 5 & Dominic & 6 & & & Dominik & 3 \\
\hline & & & & & & Dominikus & 2 & Dominikus & 2 \\
\hline & & & & & & Dominicus & 4 & Dominicus & 3 \\
\hline
\end{tabular}

Tabelle 16. Petricioli — Dominik

\begin{tabular}{|l|r|l|l|l|r|}
\hline \multicolumn{2}{|c|}{ KROATISCH -2001} & \multicolumn{2}{c|}{ ENGLISCH -1997} & \multicolumn{2}{|c|}{ DEUTSCH - 1997 } \\
\hline Dominik & 2 & Dominic & 2 & Dominik & 2 \\
\hline
\end{tabular}

In kroatischen Reiseführern wird das Endonym Dominik systematisch angeführt, während in englischsprachigen Reiseführern das Exonym Dominic verwendet wird.

In deutschsprachigen Reiseführern von Travirka werden sowohl die exonymischen Varianten Dominikus und Dominicus als auch die endonymische Variante Dominik benutzt, während bei Petricioli systematisch das Endonym Dominik verwendet wird.

In den Broschüren wird der Heiligenname Dominik nicht angeführt. 
Im Wörterbuch MG wird der Name Dominik als Dominikus übersetzt. In dem Wörterbuch KW und auf URL1 wird der Name Dominic verwendet, während im H keine Variante erscheint.

\subsection{Kuzma i Damjan}

Tabelle 17. Travirka — Kuzma i Damjan

\begin{tabular}{|c|c|c|c|c|c|c|c|c|c|}
\hline \multirow{2}{*}{\multicolumn{2}{|c|}{$\begin{array}{c}\text { KROATISCH } \\
2003\end{array}$}} & \multicolumn{4}{|c|}{ ENGLISCH } & \multicolumn{4}{|c|}{ DEUTSCH } \\
\hline & & \multicolumn{2}{|l|}{1998} & \multicolumn{2}{|l|}{2010} & \multicolumn{2}{|l|}{1998} & \multicolumn{2}{|l|}{2010} \\
\hline $\begin{array}{c}\text { Kuzma } \\
\text { i Damjan }\end{array}$ & 1 & $\begin{array}{c}\text { Cosimus and } \\
\text { Damianus }\end{array}$ & 1 & $\begin{array}{c}\text { Cosimus and } \\
\text { Damianus }\end{array}$ & 1 & $\begin{array}{c}\text { Thomas und } \\
\text { Damianus }\end{array}$ & 1 & $\begin{array}{c}\text { Thomas und } \\
\text { Damianus }\end{array}$ & 1 \\
\hline
\end{tabular}

Tabelle 18. Petricioli — Kuzma i Damjan

\begin{tabular}{|l|c|c|c|c|c|}
\hline \multicolumn{2}{|c|}{ KROATISCH - 2001 } & \multicolumn{2}{c|}{ ENGLISCH - 1997 } & \multicolumn{2}{c|}{ DEUTSCH - 1997 } \\
\hline Kuzma i Damjan & 1 & Kuzma and Damian & 1 & Kosmas und Damian & 1 \\
\hline
\end{tabular}

In beiden kroatischsprachigen Reiseführern werden die endonymischen Heiligennamen Kuzma und Damjan angeführt.

In Travirkas englischsprachigen Reiseführern werden die exonymischen Heiligennamen Cosimus und Damianus verwendet, während Petricioli einen endonymischen (Kuzma) und einen exonymischen (Damian) kombiniert.

In Travirkas (1998 und 2010) deutschsprachigen Reiseführern werden die Namen Thomas und Damianus angeführt, wobei das Exonym Thomas nicht dem Endonym Kuzma entspricht.

In den Broschüren werden die Heiligennamen Kuzma und Damjan nicht angeführt.

In den Wörterbüchern KW und H wurden keine Varianten der Namen gefunden, während URL1 und URL2 die exonymischen Varianten Cosmas und Damian gebrauchen.

\subsection{Dimitrij}

Tabelle 19. Travirka — Dimitrij

\begin{tabular}{|c|c|c|c|c|c|c|c|c|c|}
\hline \multicolumn{2}{|c|}{ KROATISCH } & \multicolumn{4}{c|}{ ENGLISCH } & \multicolumn{3}{c|}{ DEUTSCH } \\
\hline \multicolumn{2}{|c|}{2003} & \multicolumn{2}{|c|}{1998} & \multicolumn{2}{c|}{2010} & \multicolumn{2}{c|}{1998} & \multicolumn{2}{c|}{2010} \\
\hline Dimitrij & 1 & Demetrius & 1 & Demetrius & 2 & Dimitros & 1 & Demetrius & 2 \\
\hline & & & & Demetriou & 1 & & & & \\
\hline
\end{tabular}


Tabelle 20. Petricioli — Dimitrij

\begin{tabular}{|c|c|c|c|c|c|}
\hline \multicolumn{2}{|c|}{ KROATISCH - 2001 } & \multicolumn{2}{c|}{ ENGLISCH - 1997 } & \multicolumn{2}{c|}{ DEUTSCH - 1997 } \\
\hline Dimitrij & 1 & Dimitrius & 1 & Demetrius & 1 \\
\hline
\end{tabular}

In kroatischsprachigen Reiseführern von Travirka und Petricioli wird das Endonym Dimitrij verwendet.

In Travirkas englischsprachigen Reiseführern erscheinen zwei Exonyme Demetrius und Demetriou, während in Petriciolis Reiseführer das Exonym Dimitrius verwendet wird.

In Travirkas deutschsprachigen Reiseführern werden die Exonyme Dimitros und Demetrius angeführt, während der Name Demetrius auch in Petricioli vorkommt.

In den Broschüren wird der Heiligenname Dimitrij nicht angeführt.

In den Wörterbüchern MG und KW wurden keine Varianten des Namens gefunden, während URL1 und H das Exonym Demetrius anführen.

\subsection{Andere Heiligennamen}

Tabelle 21. Travirka — andere Heiligennamen

\begin{tabular}{|l|c|l|c|l|c|l|l|l|c|}
\hline \multicolumn{2}{|c|}{ KROATISCH } & \multicolumn{5}{|c|}{ ENGLISCH } & \multicolumn{3}{c|}{ DEUTSCH } \\
\hline \multicolumn{2}{|c|}{2003} & \multicolumn{2}{|c|}{1998} & \multicolumn{2}{c|}{2010} & \multicolumn{2}{c|}{1998} & \multicolumn{2}{c|}{2010} \\
\hline Martin & 2 & Martin & 4 & Martin & 4 & Martin & 3 & Martin & 3 \\
\hline & & $\begin{array}{l}\text { John } \\
\text { the Baptist }\end{array}$ & 1 & $\begin{array}{l}\text { John } \\
\text { the Baptist }\end{array}$ & 1 & $\begin{array}{l}\text { Johannes } \\
\text { der Täufer }\end{array}$ & 1 & & \\
\hline Ilija & 4 & Elias & 3 & Elias & 4 & Elias & 2 & Elias & 4 \\
\hline Toma & 4 & Thomas & 4 & Thomas & 4 & Thomas & 3 & Hl. Thomas & 4 \\
\hline Grgur & 1 & Gregory & 1 & Gregory & 1 & Gregorius & 1 & Gregorius & 1 \\
\hline Jeronim & 1 & & & Jerome & 1 & & & Hyeronymos & 1 \\
\hline Lovre & 4 & Lawrence & 4 & Lawrence & 3 & Lorenz & 4 & Lorenz & 3 \\
\hline Ante & 1 & Anthony & 1 & Anthony & 1 & & & Antonius & 1 \\
\hline Nikola & 2 & Nicholas & 2 & Nicholas & 2 & Nikolaus & 2 & Nikolaus & 2 \\
\hline Andrija & 4 & Andrew & 6 & Andrew & 4 & Andreas & 5 & Andreas & 2 \\
\hline $\begin{array}{l}\text { Petar } \\
\text { Stari }\end{array}$ & 4 & $\begin{array}{l}\text { Peter } \\
\text { the Old }\end{array}$ & 4 & $\begin{array}{l}\text { Peter } \\
\text { the Old }\end{array}$ & 3 & $\begin{array}{l}\text { Petrus } \\
\text { der Alte }\end{array}$ & 5 & $\begin{array}{l}\text { Petrus } \\
\text { der Alte }\end{array}$ & 4 \\
\hline Roko & 3 & Roch & 3 & Roch & 3 & Rocus & 2 & Rocus & 2 \\
\hline Stjepan & 4 & Stephen & 2 & Stephen & 3 & Stephanus & 2 & & \\
\hline Mihovil & 2 & Michael & 4 & Michael & 2 & Michael & 2 & Michael & 2 \\
\hline
\end{tabular}


Tabelle 22. Petricioli — andere Heiligennamen

\begin{tabular}{|l|c|l|c|l|c|}
\hline \multicolumn{2}{|c|}{ KROATISCH - 2001 } & \multicolumn{2}{c|}{ ENGLISCH - 1997 } & \multicolumn{2}{c|}{ DEUTSCH - 1997 } \\
\hline Martin & 4 & Martin & 2 & Martin & 4 \\
\hline Ivan Krstitelj & 1 & John the Baptist & 1 & Johannes der Täufer & 1 \\
\hline Ilija & 3 & Elia & 2 & $\begin{array}{l}\text { Elias } \\
\text { Elias (Ilija) }\end{array}$ & 2 \\
\hline & 4 & Thomas & 2 & Thomas & 2 \\
\hline Grgur & & & & Thomas (Toma) & 1 \\
\hline Jeronim & 1 & Grgur & 1 & Gregor & 1 \\
\hline Lovre & 7 & Jeronimo & 4 & Hieronymus & 6 \\
\hline & 3 & Lovro (Lawrence) & 1 & Lauentius & 2 \\
\hline Antun & & Lovro & 1 & Laurentius (Lovro) & 1 \\
\hline Nikola & 2 & Anthony & 1 & Antonius & 2 \\
\hline Andrija & 4 & Nicholas & 1 & Nikolaus & 4 \\
\hline Petar Stari & 3 & Andrew & 7 & Andreas & 9 \\
\hline & & Peter the Old & 1 & Petar Stari & 2 \\
\hline Roko & 4 & Rocco & 1 & & \\
\hline Stjepan & 3 & Stephen & 2 & Rochus & 3 \\
\hline Mihovil & 4 & Michael & 2 & Michael & 3 \\
\hline & & & & Michael (Mihovil) & 1 \\
\hline
\end{tabular}

In allen untersuchten Reiseführern erscheint der Name Martin.

Die folgenden Heiligennamen werden konsequent verwendet: John the Baptist Johannes der Täufer, Toma - Thomas - Thomas, Nikola - Nicholas - Nikolaus, Andrija - Andrew - Andreas und Mihovil - Michael - Michael.

Der folgende Heiligenname hat zwei endonymische Varianten, während die englischen und deutschen Exonyme systematisch vorkommen: Ante (Travirka)/Antun (Petricioli) — Anthony - Antonius.

In den untersuchten Wörterbüchern und URL-Seiten werden die oben genannten Varianten der Namen verwendet.

In den folgenden Heiligennamen wird anstatt einer Übersetzung ein Endonym angeführt: Grgur — Gregory (Travirka)/Grgur (Petricioli) — Gregorius (Travirka)/ Gregor (Petricioli), Lovre — Lawrence (Travirka)/Lovro (Petricioli) — Lorenz (Travirka)/Laurenzius (Petricioli) und Petar Stari - Peter the Old - Petrus der Alte (Travirka)/Petar Stari (Petricioli).

In den folgenden Heiligennamen unterscheiden sich die exonymischen Einträge zwischen den Reiseführern von Travirka und Petricioli: Ilija - Elias (Travirka)/Elia (Petricioli) — Elias, Grgur — Gregory (Travirka)/Grgur (Petricioli) — Gregorius (Travirka)/Gregor (Petricioli), Jeronim — Jerome (Travirka)/Jeronimo (Petricioli) - Hyeronymos (Travirka)/Hieronymus (Petricioli), Lovre - Lawrence (Travirka)/ 
Lovro (Petricioli) - Lorenz (Travirka)/Laurenzius (Petricioli), Roko - Roch (Travirka)/Rocco (Petricioli) — Rocus (Travirka)/Rochus (Petricioli) und Stjepan Stephen - Stephanus (Travirka)/Stephan (Petricioli).

In den folgenden Wörterbüchern und URL-Seite erscheinen:

MG: Elia, Elias, Elija; Gregor; Hieronymus; Laurentius; Rochus; Stephan, Stephanus.

KW: Elias; Gregory, Jerome.

H: Elias, Stephen.

URL1: Elias, Gregory, Jerome, Lawrence, Roch, Stephen.

\section{SCHLUSSFOLGERUNG}

Dieser Beitrag hat als Ziel, einen Vorschlag für einen systematischen Gebrauch der Heiligennamen in Reiseführern und Broschüren von Zadar auszuarbeiten. Demzufolge wurde auf die folgenden Fragen geantwortet: Wird ein Name systematisch verwendet oder wird er mit mehreren Varianten kombiniert? Werden in den englischsprachigen und deutschsprachigen Reiseführern und Broschüren endonymische oder exonymische Varianten der Namen verwendet?

Die Untersuchung ergab zahlreiche Unregelmäßigkeiten in der Verwendung der Heiligennamen. In den englischsprachigen und deutschsprachigen Reiseführern und Broschüren kommen abwechselnd endonymische und exonymische Varianten der Namen vor.

Unser Vorschlag ist, dass sich die Autoren und Übersetzer der Reiseführer und Broschüren bei der Anwendung der Heiligennamen für eine exonymische Variante entscheiden sollten und diese Variante systematisch verwenden. Es ist auch wünschenswert, dass bei dem ersten Auftreten des Heiligennamens das Endonym in Klammern angeführt wird, damit auch die Touristen mit den einheimischen Heiligennamen vertraut werden.

\section{LITERATURVERZEICHNIS}

Primärliteratur

discover... Zadar, 2016, Zadar Tourist Board, Zadar. entdecke... Zadar, 2016, Fremdenverkehrsamt der Stadt Zadar, Zadar.

otkrij... Zadar, 2016, Turistička zajednica grada Zadra, Zadar.

Petricioli I. 1997: Zadar and its Surroundings: History, culture, art, natural features, tourism, plan of the city and its surroundings, Turistička naklada, Zagreb.

Petricioli I. 1997: Zadar und Umgebung: Geschichte, Kultur, Kunst, Naturschönheiten, Tourismus, Stadtplan, Landkarte, Turistička naklada, Zagreb.

Petricioli I. 2001: Zadar i okolica: Povijest, kultura, umjetnost, prirodne ljepote, Turistička naklada, Zagreb. 
Travirka A. 1998: Zadar. Geschichte. Kultur. Künstlerisches Erbe, Forum, Zadar.

Travirka A. 1998: Zadar. History. Culture. Art Heritage, Forum, Zadar.

Travirka A. 2003: Zadar. Povijest. Kultura. Umjetnička baština, Forum, Zadar.

Travirka A. 2010: Zadar. Geschichte. Kultur. Künstlerisches Erbe, Forum, Zadar.

Travirka A. 2010: Zadar. History. Culture. Art Heritage, Forum, Zadar.

\section{Sekundärliteratur}

Duden. 2003: Deutsches Universalwörterbuch. Dudenverl., Mannheim.

Frančić A. 2015: Suvremena hrvatska onomastika - izazovi, potrebe i mogućnosti, „Croatica“ XXXIX, 59, Zagreb, 75-85.

Harder K. B. 1995: Names in Language Contact: Exonyms, In: Eichler E. u.a, (hrsg.), Namenforschung: Ein internationales Handbuch zur Onomastik / Name Studies: An International Handbook of Onomastics / Les noms propers: Manuel international d'onomastique, Walter de Gruyter, Berlin-New York.

Hitchcock R. D. 1869: Hitchcock's New and Complete Analysis of the Holy Bible, A. J. Johnson, New York.

Kessler E., Wenborn N. 2005: A Dictionary of Jewish-Christian Relations, Cambridge University Press, Cambridge-New York.

Matasović R. 1997: O metodologiji onomastičkih istraživanja (na primjeru keltske onomastike), „Folia onomastica Croatica“" 6, S. 89-98.

Mikić P., Gojmerac M. 2011: Hrvatsko-njemački religijski rječnik. Religionswörterbuch KroatischDeutsch, Naklada Slap, Jastrebarsko.

Šimunović P. 2009: Uvod u hrvatsko imenoslovlje, Golden marketing — Tehnička knjiga, Zagreb.

URL1 $=$ www.catholic.org $(20.8 .2016)$

URL2 $=$ www.americancatholic.org (20.8.2016)

\section{NAMES OF SAINTS IN TOURIST BROCHURES AND TOUR GUIDES IN ZADAR}

\section{SUMMARY}

Zadar is one of the oldest cities in Croatia. It has a 3000 years old history. In the fourth century, when Christianity spread, not only in Zadar, but in whole Croatia, the construction of the first churches began. Nowadays, religion still plays an important role in Zadar's cultural heritage, as Croatia is a predominantly Catholic country. Zadar has four patron saints (Saint Stošija, Saint Zoilo, Saint Šime and Saint Krševan), and one can find over 30 churches in the centre of Zadar alone. Because of the turbulent history, mostly in World War II and the Homeland War, a great number of churches were destroyed, but the names of the saints are still remembered and worshipped. Because of Zadar's role in tourism, it is necessary that these names be properly and consistently translated. In the most popular and the most distributed tourist brochures and guides in Zadar, these names are not used in their endonymic form, but are translated. The problem occurs when there are inconsistencies in these translations.

This research will throw light upon this problematic usage of endonymic and exonymic forms. An endonymic form of the saints' names is the original name, which is used in Croatia, whereas an exonymic form is the one used in other languages. The research will include the textual analysis of the names of saints in Zadar. The aim of this research is to find appropriate exonymic equivalents for endonymic names of saints in order to improve and propose consistency to the tourist brochures and guides in German and English.

Keyw ord s: saints' names, tourist guides and brochures, endonyms, exonyms 